\title{
Non-invasive imaging of neuropathology in a rat model of $\alpha$-synuclein overexpression
}

\author{
Erwin Lauwers ${ }^{\mathrm{a}}$, Dirk Bequé ${ }^{\mathrm{b}}$, Koen Van Laere ${ }^{\mathrm{b}}$, Johan Nuyts ${ }^{\mathrm{b}}$, Guy Bormans ${ }^{\mathrm{c}}$, \\ Luc Mortelmans $^{\mathrm{b}}$, Cindy Casteels ${ }^{\mathrm{b}}$, Linda Vercammen ${ }^{\mathrm{a}}$, Olivier Bockstael ${ }^{\mathrm{d}}$, \\ Bart Nuttin ${ }^{\mathrm{e}}$, Zeger Debyser ${ }^{\mathrm{f}, \mathrm{g}}$, Veerle Baekelandt ${ }^{\mathrm{a}}$,* \\ a Laboratory for Neurobiology and Gene Therapy, K.U. Leuven, Leuven, Belgium \\ ${ }^{\mathrm{b}}$ Division of Nuclear Medicine, K.U. Leuven, Leuven, Belgium \\ ${ }^{\mathrm{c}}$ Laboratory for Radiopharmaceutical Chemistry, K.U. Leuven, Leuven, Belgium \\ ${ }^{\mathrm{d}}$ Laboratory of Experimental Neurosurgery, Institute of Interdisciplinary Research, Faculty of Medicine, \\ Université Libre de Bruxelles, Belgium \\ e Experimental Neurosurgery and Neuroanatomy, K.U. Leuven, Leuven, Belgium \\ ${ }^{\mathrm{f}}$ Laboratory for Molecular Virology and Gene Therapy, K.U. Leuven, Leuven, Belgium \\ ${ }^{\mathrm{g}}$ K.U. Leuven Campus Kortrijk, Kortrijk, Belgium
}

Received 15 July 2005; received in revised form 24 November 2005; accepted 6 December 2005

Available online 19 January 2006

\begin{abstract}
Parkinson's disease is a neurodegenerative disorder affecting the dopaminergic neurons in the substantia nigra. Aggregation of $\alpha$-synuclein appears to play a central role in the pathogenesis. Novel animal models for neurodegeneration have been generated by lentiviral vector-mediated locoregional overexpression of disease-associated genes in the adult brain. We have used lentiviral vectors to overexpress a clinical mutant of $\alpha$-synuclein, A30P, in the rat substantia nigra. This overexpression induced time-dependent cytoplasmic and neuritic accumulation of $\alpha$-synuclein and neurodegeneration. A subgroup of the rats developed asymmetric rotational behavior after administration of amphetamine. In addition, these animals displayed reduced dopamine transporter binding visualized by ${ }^{123}$ I-FP-CIT microSPECT imaging. The behavioral and microSPECT data were validated by histological analysis. There was a strong correlation between the reduction of dopaminergic neurons in the substantia nigra and the reduction of dopamine transporter binding in the striatum. MicroSPECT imaging enables non-invasive imaging of the neurodegeneration allowing longitudinal follow-up in this new animal model for Parkinson's disease and the evaluation of neuroprotective drugs.
\end{abstract}

(C) 2005 Elsevier Inc. All rights reserved.

Keywords: Parkinson's disease; $\alpha$-Synuclein; Neurodegeneration; Animal model; SPECT

Abbreviations: PD, Parkinson's disease; 6OHDA, 6-hydroxydopamine; MPTP, 1-methyl-4-phenyl-1,2,3,6-tetra-hydropyridine; GFP, green fluorescent protein; $\alpha$-SYN, $\alpha$-synuclein; WPRE, Woodchuck hepatitis post-transcriptional regulatory element; cPPT, central polypurine tract; VSV, vesicular stomatitis virus; 123I-FP-CIT, ${ }^{123} \mathrm{I}-\mathrm{N}$ - $\omega$-fluoropropyl-2- $\beta$ carboxymethoxy-3 $\beta$-(4-iodophenyl); SPECT, single-photon emission computed tomography; VOI, volume-of-interest; TH, tyrosine hydroxylase; $\mathrm{LV}$, lentiviral vector; $\mathrm{SN}$, substantia nigra; $\mathrm{AAV}$, adeno-associated virus; PGK, phosphoglycerate kinase; MRI, magnetic resonance imaging; PEI, polyethylenimine; DAB, diaminobenzidine

* Corresponding author at: Kapucijnenvoer 33, VCTB +5, B-3000 Leuven, Belgium. Tel.: +32 163321 56; fax: +32163363 36 .

E-mail address: veerle.baekelandt@med.kuleuven.be (V. Baekelandt).

\section{Introduction}

Aggregation of $\alpha$-synuclein is a key event in the pathogenesis of Parkinson's disease (PD). $\alpha$-Synuclein was first linked to Parkinson's disease by the discovery of two point mutations (A53T and A30P) in the $\alpha$-synuclein gene associated with familial forms of PD $[19,26]$. Later, $\alpha$-synuclein was identified as the major component of Lewy bodies and Lewy neurites, the neuropathological hallmarks of PD [30]. More recently, a third point mutation (E46K) was identified as a cause of hereditary PD [34]. Purified $\alpha$-synuclein is 
known to form protein aggregates in vitro under certain conditions $[9,13]$. Several animal models for Parkinson's disease have been developed. These models play an important role in the understanding of the pathogenesis of Parkinson's disease and in the development of new therapies. The first animal models were based on the acute destruction of dopaminergic neurons using selective neurotoxins, which include 6-hydroxydopamine (6OHDA) and 1-methyl-4-phenyl1,2,3,6-tetra-hydropyridine (MPTP) [3]. More recently, transgenic mice and fruit flies overexpressing wild type or mutant $\alpha$-synuclein have been developed [10,14,22,27,32]. The $\alpha$-synuclein transgenic mice display varying degrees of brain pathology, but they do not display specific neurodegeneration of the dopaminergic neurons or dopaminedependent motor impairment, both characteristic of PD. In Drosophila, human wild type and mutant (A30P and A53T) $\alpha$-synuclein were expressed in neurons. These transgenic flies develop a progressive age-dependent locomotor dysfunction, accompanied by a selective loss of dopaminergic neurons.

Recently, we and others have developed alternative models based on local viral vector-mediated overexpression of $\alpha$ synuclein. Recombinant lentiviral (LV) and adeno-associated virus (AAV) vectors have been used for the targeted overexpression of both wild type and mutant $\alpha$-synuclein in the rat substantia nigra $[6,17,18]$. These rats develop chronic progressive Lewy pathology and neurodegeneration of the dopaminergic neurons.

We have previously reported progressive neuropathological changes and neurodegeneration after LV-mediated overexpression of $\alpha$-synuclein in different regions of the mouse brain [20]. One of the major challenges of the viral vectorbased models is the possibility to monitor neurodegeneration in a non-invasive way over time to allow direct correlation with behavioral performance and to test new potential therapeutic agents for PD.

We here report a long-term follow-up study of a group of rats that were injected in the substantia nigra with $\mathrm{LV}$ encoding A30P $\alpha$-synuclein ( $\mathrm{LV}-\alpha-\mathrm{SYN}(\mathrm{A} 30 \mathrm{P}))$. We validated this rat model by behavioral testing, non-invasive molecular imaging and histological analysis. The chronic progressive nature of the pathology and the non-invasive microSPECT (single-photon emission computed tomography) imaging both will facilitate the evaluation of novel treatment strategies targeting the synucleinopathy in this model.

\section{Materials and methods}

\subsection{Animals}

We used adult Wistar rats in this study. The animals were housed under $14 \mathrm{~h}$ light $/ 10 \mathrm{~h}$ dark cycle with free access to food and water. All animal experiments were approved by the bioethical committee of the K.U. Leuven.

\subsection{Lentiviral vector construction and production}

The cDNA encoding the human clinical mutant (A30P) of $\alpha$-synuclein, obtained from Dr. Kelly Conway (Center for Neurologic Diseases, Boston), was cloned into the pCHMWS and the pHMWS transfer plasmids. These plasmids are derived from the original pHR transfer plasmid [23]. A multiple cloning site and a self-inactivating deletion in the $\mathrm{U} 3$ region (pHR'SIN-18) were created [37]. Additionally, the Woodchuck hepatitis post-transcriptional regulatory element (WPRE) [36] and the central polypurine tract (cPPT or C) $[11,35]$, respectively, were inserted. HIV-1-derived vector particles, pseudotyped with the envelope of vesicular stomatitis virus (VSV), were produced by transfecting 293T cells with a packaging plasmid encoding viral Gag and Pol proteins (pCMVDR8.91), a plasmid encoding the envelope of vesicular stomatitis virus (pMDG), and the transfer plasmid encoding $\alpha$-synuclein. Transient transfection of 293T cells was carried out in $10-\mathrm{cm}$ dishes. For each plate, a DNA mixture (700 ml) containing $20 \mathrm{mg}$ of transfer plasmid, $10 \mathrm{mg}$ of packaging construct, and $5 \mathrm{mg}$ of envelope plasmid in $150 \mathrm{mM}$ $\mathrm{NaCl}$ was prepared. A 700-ml volume of polyethylenimine (PEI) solution $(110 \mathrm{ml}$ of $10 \mathrm{mM}$ PEI stock solution, $\mathrm{pH}$ 7.0 , diluted with $590 \mathrm{ml}$ of $150 \mathrm{mM} \mathrm{NaCl}$ ) was added to this DNA mixture. After $15 \mathrm{~min}$ at room temperature, the DNA-PEI complex was added dropwise to the 293T cells in Opti-MEM I (GIBCO-BRL, Merelbeke, Belgium) with gentamicin $(20 \mathrm{mg} / \mathrm{ml})$ and without fetal calf serum (FCS). After overnight incubation, the medium was changed. Supernatants were collected from days 2 to 5 post-transfection. The vector particles in the supernatant were filtered through a $0.45 \mu \mathrm{m}$ pore size filter and then sedimented by ultracentrifugation at $15,000 \mathrm{rpm}$ at $4{ }^{\circ} \mathrm{C}$ for $5 \mathrm{~h}$. The pellets were redissolved in phosphate-buffered saline (PBS). For in vivo experiments, the vectors were further centrifuged for $1 \mathrm{~h}$ at $20,000 \mathrm{rpm}$ and redissolved in PBS, resulting in a final 8000-fold concentration. p24 Antigen content was determined with the HIV-1 p24 core profile ELISA (Perkin-Elmer Life Sciences, Boston, MA).

\subsection{Surgery}

We performed all surgical procedures under ketamine $(60 \mathrm{mg} / \mathrm{kg}$ i.p.) and medetomidine $(0.4 \mathrm{mg} / \mathrm{kg})$ anaesthesia using aseptic procedures. The rats were placed in a stereotactic head frame (Stoelting, Wood Dale, IL) and a small hole was drilled in the skull in the appropriate location using bregma as reference. The coordinates used to target the substantia nigra were: AP -5.2, L 2.3 and DV 7.3. We injected $4 \mu \mathrm{l}$ of highly concentrated vector $\left(10^{8}-10^{9} \mathrm{pg} \mathrm{p} 24 / \mathrm{ml}\right)$ supplemented with $4 \mu \mathrm{g} / \mathrm{ml}$ polybrene at a rate of $0.25 \mu \mathrm{l} / \mathrm{min}$. After the injection, the needle was left in place for an additional $10 \mathrm{~min}$ before being slowly withdrawn from the brain.

6OHDA lesions were performed by unilateral injection of $24 \mu \mathrm{g}$ 6-hydroxydopamine (6OHDA; Sigma, St. QuentinFallavier, France) dissolved in $3 \mu \mathrm{l}$ of $0.9 \%$ sterile $\mathrm{NaCl}$ 
containing $0.1 \%$ ascorbic acid in the substantia nigra. The coordinates used were: AP -5.2, L 2.1 and DV 7.2.

\subsection{Behavioral testing}

Behavioral testing was performed on two groups of 15 rats.

In the stepping test, the animals were tested for forelimb akinesia [16]. Two days before the test, the rats were familiarized with the test procedure. The test was performed twice daily on three consecutive days by an experimenter unaware of the group identity of the tested rats resulting in total six registrations for each animal in each direction. The rat was held by the experimenter fixing both hind limbs with one hand and the forelimb not to be monitored with the other hand. The unrestrained forepaw was touching the table and the number of adjusting steps was counted while the rat was moved sideways along the table surface in the direction of the extended paw $(90 \mathrm{~cm}$ in $5 \mathrm{~s})$. This procedure was repeated twice for each forelimb and the mean of data obtained on three testing days constituted the final value. At different time points after injection, amphetamine-induced asymmetric rotational behavior was monitored in an automated rotometer bowl [31]. Amphetamine was injected intraperitoneally at a dose of $5 \mathrm{mg} / \mathrm{kg}$. For each test the total number of complete turns clockwise and counter-clockwise was counted over $90 \mathrm{~min}$. The direction ipsilateral to the lesion is considered as positive. The analysis of amphetamine tests was based on net ipsilateral turns (defined as clockwise turning in case of a right-sided injection).

\subsection{Molecular imaging and analysis}

Five Wistar rats 9 months after unilateral transduction with a LV encoding A30P $\alpha$-synuclein, four 6OHDA lesioned rats and five normal age-matched rats were injected with $300 \mathrm{MBq}$ 123I-FP-CIT ( ${ }^{123} \mathrm{I}-\mathrm{N}-\omega$-fluoropropyl-2- $\beta$ carboxymethoxy-3 $\beta$-(4-iodophenyl) nortropane) to evaluate the dopamine transporters in the rat striatum.

Ninety minutes after injection, the rats were anaesthetized with nembutal $(60 \mathrm{mg} / \mathrm{kg})$ and microSPECT was carried out using a single-head Millennium GE gamma camera with a $3 \mathrm{~mm}$ aperture single-pinhole collimator. Images were acquired in a $128 \times 128$ matrix over 64 projection angles in step-and-shoot mode, frame duration $60 \mathrm{~s}$ per angle. The pinhole geometry was determined using an in-house developed algorithm [4] and tomographic images were produced with maximum-likelihood reconstruction (the equivalent of 600 iterations was applied, accelerated using subsets, with a gradually decreasing number of subsets per iteration). During reconstruction, the position dependent resolution was modeled by tracing seven rays through the pinhole aperture per detector pixel. Attenuation was ignored. The voxel size after reconstruction is $1.2 \mathrm{~mm} \times 1.2 \mathrm{~mm} \times 1.2 \mathrm{~mm}$, the spatial resolution was about $3 \mathrm{~mm}$. Reconstructed images were quantified by volume-of-interest (VOI) analysis (PMOD Software v2.5, Zurich, Switzerland). VOI contours were based on a predefined structural template (volume striatum VOI $0.042 \mathrm{~cm}^{3}$ ) and manually adjusted per animal (translation only). The average voxel size of the striatal VOIs in this study was $55 \pm 4$ (1 S.D.) voxels, for the cerebellum (three sections) on average $42 \pm 3$ voxels were taken. For semiquantification, the binding index (striatum/cerebellum - 1) for left and right striatal volumes-of-interest was calculated and right-to-left binding asymmetry indices were determined as $R / L$ by comparison to the unaffected left side.

\subsection{Histology}

To assess lentiviral transduction, we deeply anaesthetized the rats with pentobarbital and perfused them transcardially with saline followed by ice-cold $4 \%$ paraformaldehyde in PBS for $15 \mathrm{~min}$. The brain was postfixed overnight in the same fixing solution. $50 \mu \mathrm{m}$-thick coronal brain sections were cut with a vibratome and stored at $4{ }^{\circ} \mathrm{C}$. First, sections were treated with $3 \%$ hydrogen peroxide and incubated overnight with the primary rabbit anti- $\alpha$-synuclein antibody (1:5000) (Chemicon, Temecula, CA) or rabbit anti-tyrosine hydroxylase (1:5000) (Chemicon) in 10\% normal swine serum. Then the sections were incubated in biotinylated swine anti-rabbit secondary antibody, followed by incubation with StreptABC-HRP complex (DAKO, Glostrup, Denmark). Detection was with diaminobenzidine (DAB) using $\mathrm{H}_{2} \mathrm{O}_{2}$ as a substrate.

For immunofluorescence, sections were incubated overnight with mouse monoclonal anti- $\alpha$-synuclein $(1: 20$, ZYMED, South San Francisco, CA) and rabbit polyclonal anti-ubiquitin (1:200, DAKO). Detection was with goat anti-mouse conjugated with Alexa-488 and goat anti-rabbit conjugated with Alexa-633 (Molecular Probes, Leiden, The Netherlands). Confocal microscopy was performed using the LSM 510-ConfoCor 2 (Zeiss).

\subsection{Quantification of nigral dopaminergic neurons}

The number of TH-positive neurons in the SN pars compacta was estimated using an unbiased stereological method: the optical fractionator (Stereo Investigator, MicroBrightField Inc.). Quantification was performed on four $\alpha-S Y N$ rats used for SPECT imaging (one of the five $\alpha-S Y N$ rats died after imaging and the brain could not be recovered), on 16 $\alpha$-SYN rats that scored negative in the amphetamine-induced rotational test, on four 6OHDA rats and on four control normal rats. Every fifth section throughout the substantia nigra was analysed with a total of seven to eight sections for each animal. A coefficient of error of $<0.10$ due to the estimation was accepted.

The volume of the transduced brain area was quantified using the Cavalieri principle as previously described [1].

\subsection{Statistical analysis}

For behavior analysis, data are presented as the mean \pm S.D. MicroSPECT semiquantitative ratios and histo- 
logical dopaminergic neurons numbers were evaluated with Statistica v6.0 (StatSoft Inc., Tulsa, USA) using the Student's $t$-test. Significance was accepted at the $95 \%$ probability level.

\section{Results}

\subsection{Lentiviral vector-mediated overexpression of GFP and $\alpha$-synuclein in the substantia nigra of the rat}

To determine the efficiency of transgene expression in the dopaminergic neurons of the rat substantia nigra, we injected LV overexpressing the green fluorescent protein (GFP). Transduction of dopaminergic neurons was verified by colocalization of GFP and tyrosine hydroxylase (TH). After a single injection of LV-GFP, produced with the transfer plasmid pCHMWS, we were able to transduce up to $85 \%$ of the dopaminergic neurons in the substantia nigra ( $\mathrm{SN}$ ) (data not shown). Next, we injected LV- $\alpha-\mathrm{SYN}(\mathrm{A} 30 \mathrm{P})$ in the substantia nigra of the rat. We compared the expression levels of $\alpha$-synuclein obtained with vectors produced with the transfer plasmids pHMWS or pCHMWS. To determine the expression levels of $\alpha$-synuclein (A30P) we quantified the transduced volume using the Cavalieri principle. In agreement with previous studies, we found that the presence of the cPPT element resulted in a two- to four-fold increase in $\alpha$ synuclein (A30P) expression in the SN (mean 3.2-fold \pm 0.9 S.D.) (data not shown) $[1,11,35]$.

\subsection{Motor behavior of rats overexpressing $\alpha$-synuclein in the substantia nigra}

To evaluate the phenotypic effect of overexpression of $\alpha$ synuclein in the substantia nigra of the rat in a dose-dependent way, we injected two groups of 15 rats with a single injection of $4 \mu$ l concentrated LV overexpressing the A30P clinical mutant of $\alpha$-synuclein. The first group was injected with LV produced with the transfer plasmid pHMWS- $\alpha-S Y N(A 30 P)$ and the second group with vectors produced with the transfer plasmid pCHMWS- $\alpha$-SYN(A30P), which will be referred to as the high expression group. In each group of 15 rats, five rats received a control injection of saline and five rats a control injection with LV-GFP in the contralateral substantia nigra. We analyzed the injected rats for possible motor dysfunction by the stepping test and the amphetamine-induced asymmetric rotational test. We started testing the rats 6 weeks after injection and retested the animals every 10 weeks. In the stepping test, no significant differences in motor performance of the two forelimbs were observed at any time point (data not shown). The amphetamine-induced asymmetric rotational behavior test is a standard test used to assess neurodegeneration in the 6OHDA lesion model for PD. A turning behavior of more than 100 ipsiversive rotations in 90 min correlates with a successful and detectable lesion in the substantia nigra. More than 500 rotations in this test is indicative of a nearly complete lesion (more than 97\%)

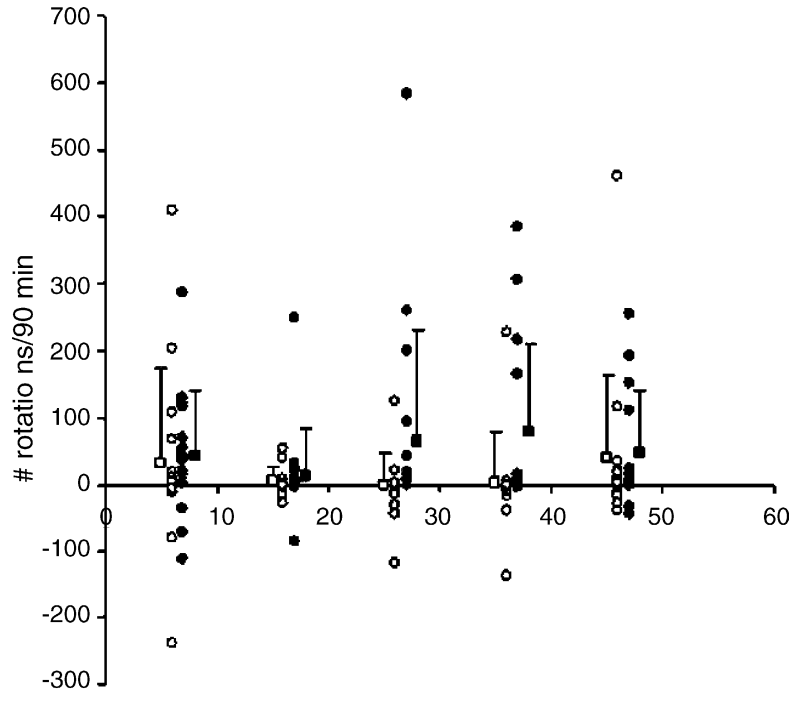

weeks

Fig. 1. Amphetamine-induced rotation test in rats transduced with $\alpha$ SYN(A30P) LV. Each circle represents the net number of ipsiversive rotations at different time points for individual rats transduced with LV produced with the transfer plasmid pHMWS (open circles) or pCHMWS (closed circles). The open en closed boxes represent the mean value \pm S.D. of the rotations at each time point.

[24]. We monitored amphetamine-induced rotational behavior until 46 weeks after injection (Fig. 1).

Six weeks after injection we observed considerable variability in rotational behavior in both groups, which disappeared again at 16 weeks. This may be due to a transient effect following injection in the substantia nigra. At later time points, a consistent ipsiversive rotational behavior was observed in the high expression group. Five rats in the high expression group showed asymmetric rotational behavior starting from week 25 . This effect was maximal at 26 weeks after injection. At later time points, the same five rats display increased rotations. Interestingly, in the group injected with the pHMWS vectors, there was no clear increase in ipsiversive rotations until 46 weeks after transduction.

\subsection{Imaging of dopamine transporter activity by ${ }^{123}$ I-FP-CIT MicroSPECT}

Since the behavioral tests pointed to a modest but significant deficit in dopaminergic neurotransmission, we wondered whether we could monitor this in a non-invasive way by microSPECT imaging of the dopamine transporter activity.

${ }^{123}$ I-FP-CIT MicroSPECT imaging was performed on 14 rats: five rats injected unilaterally with $L V-\alpha-S Y N(A 30 P)$ that performed more than 100 ipsiversive rotations induced by amphetamine at 46 weeks, four rats injected unilaterally with 6OHDA as a positive control and five age-matched control rats. In the age-matched control rats both the left and right striatum were clearly visible (Fig. 2a). In the 6OHDA rats, there was an average loss of $94 \pm 13 \%$ dopamine transporter activity (Fig. 2c) in the lesioned hemisphere as compared to the non-injected side (binding asymmetry index $R / L=0.055$ ). 


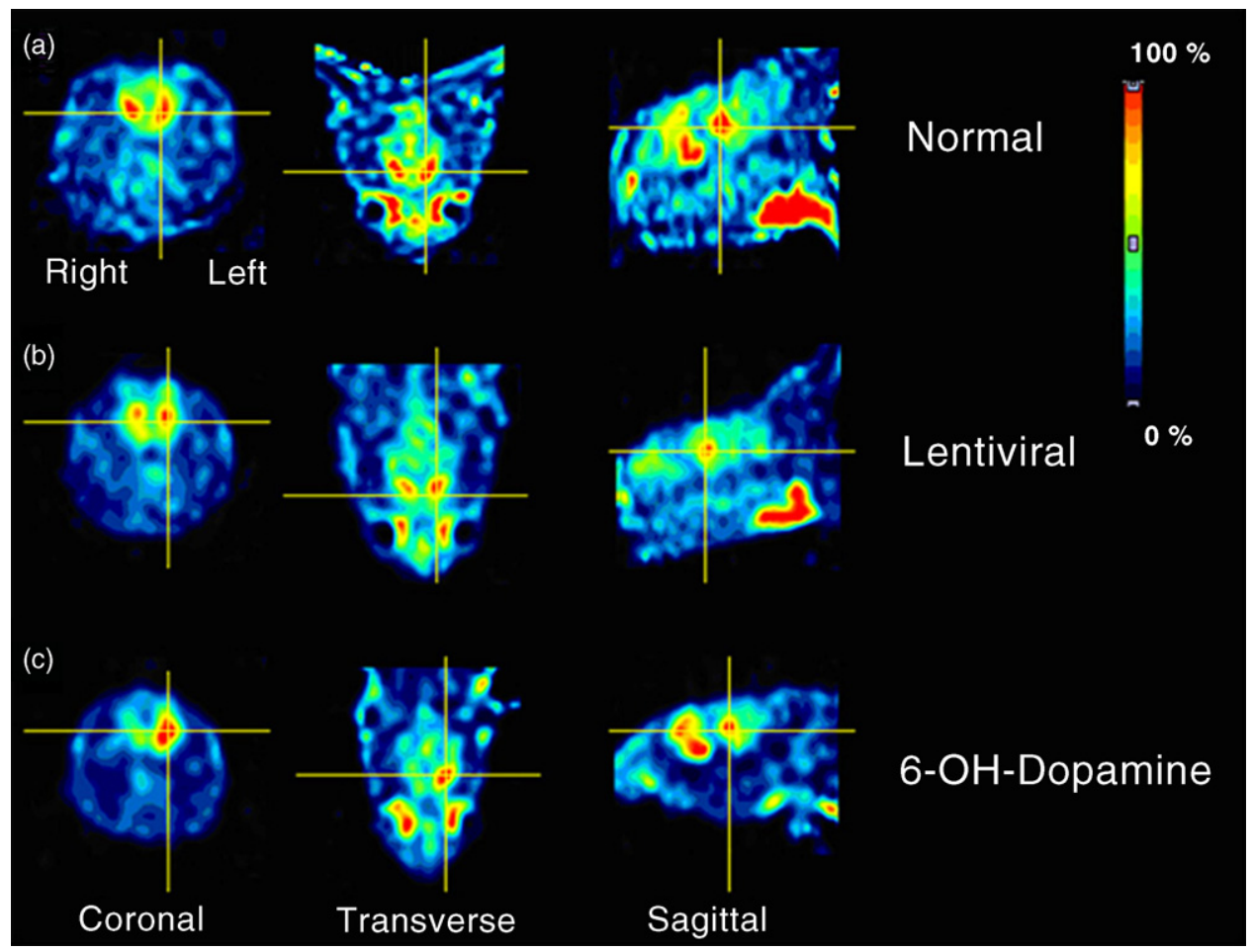

Fig. 2. ${ }^{123}$ I-FP-CIT MicroSPECT imaging. Representative images from a normal (a), a LV- $\alpha-S Y N(A 30 P)$-transduced (b) and a 6OHDA lesioned rat (c) are shown. Images show the coronal, transverse and sagittal cross-sections through the (left) striatum. The color scale (relative activity values) is given on the right.

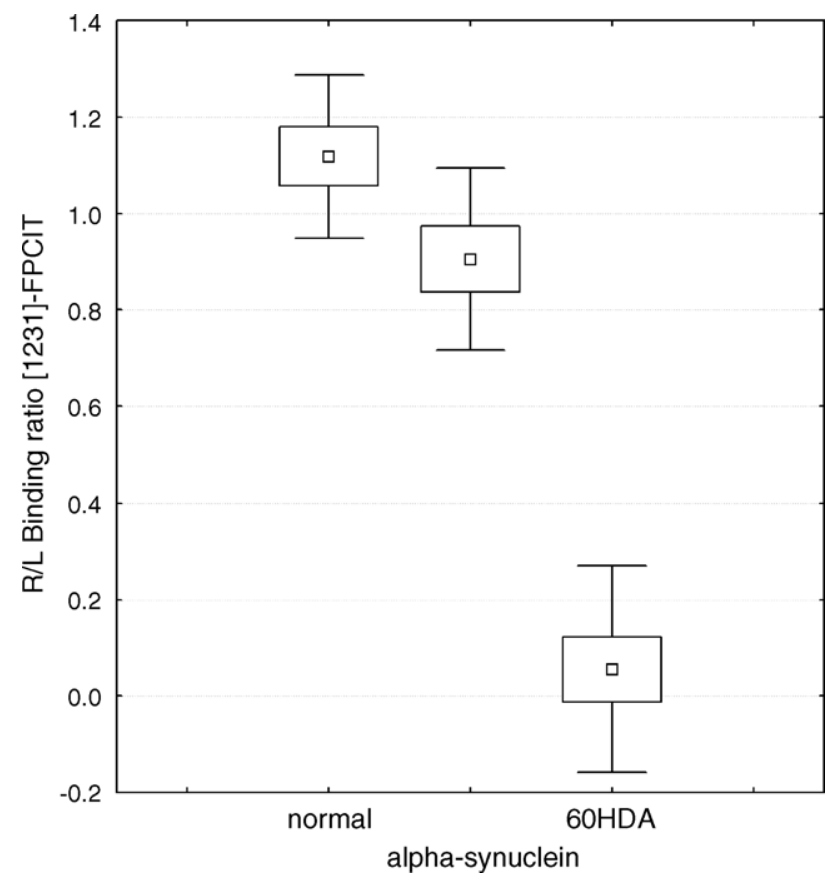

Fig. 3. Box-and-Whisker plot of the right-to-left ${ }^{123}$ I-FP-CIT binding asymmetry index. The box represents the 25 and $75 \%$ value, Whiskers denote minimum and maximum values. MicroSPECT analysis was performed 46 weeks after injection with $\mathrm{LV}-\alpha-\mathrm{SYN}(\mathrm{A} 30 \mathrm{P})$ on five rats that performed more than 100 ipsiversive rotations in the rotation test. A significant reduction in DAT was observed in the $\alpha$-synuclein overexpressing rats compared to the control rats $(n=5)(p<0.05)$. As a positive control, four 6OHDAlesioned rats were also measured.

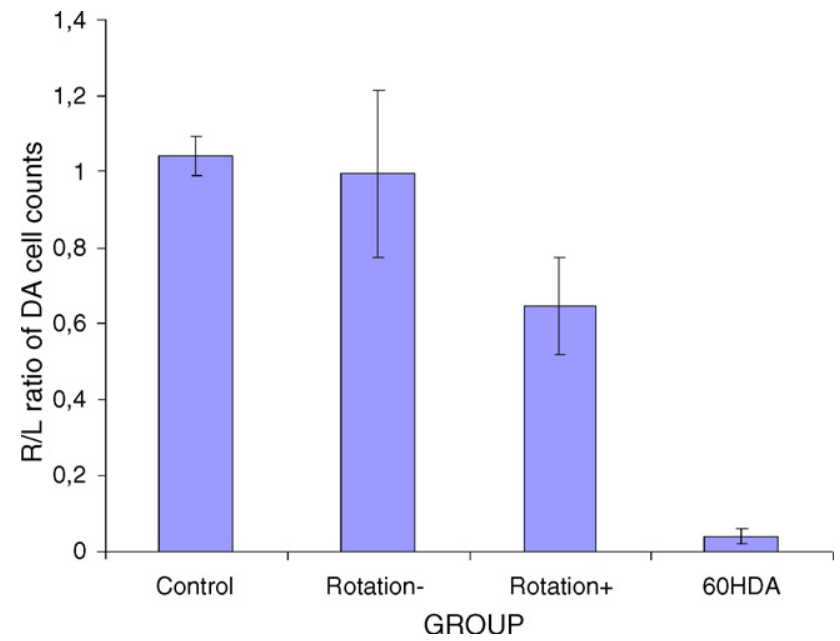

Fig. 4. Quantification of the right-to-left ratio of dopaminergic neurons in the substantia nigra determined by stereological analysis. The data are represented as the mean \pm S.D. The first group of animals are the control, non-injected rats $(n=4)$. The second group of animals are the rats injected with LV- $\alpha-S Y N(A 30 P)$ that did not display rotational behavior in the amphetamine-induced rotational test $(n=19)$. The third group of animals represents rats injected with $\mathrm{LV}-\alpha-\mathrm{SYN}(\mathrm{A} 30 \mathrm{P})$ that performed more than 100 ipsiversive rotations in the amphetamine-induced rotational test 46 weeks post-injection $(n=4)$. The last group of animals have a 6 OHDA lesion $(n=4)$. A significant difference was found between the third group of animals and the control animals $(p<0.05)$ and between the second and the third group of animals $(p<0.05)$. We also found a significant difference between the last group and all the other groups. No significant difference was found between the control group of rats and the rats that did not display rotational behavior. 

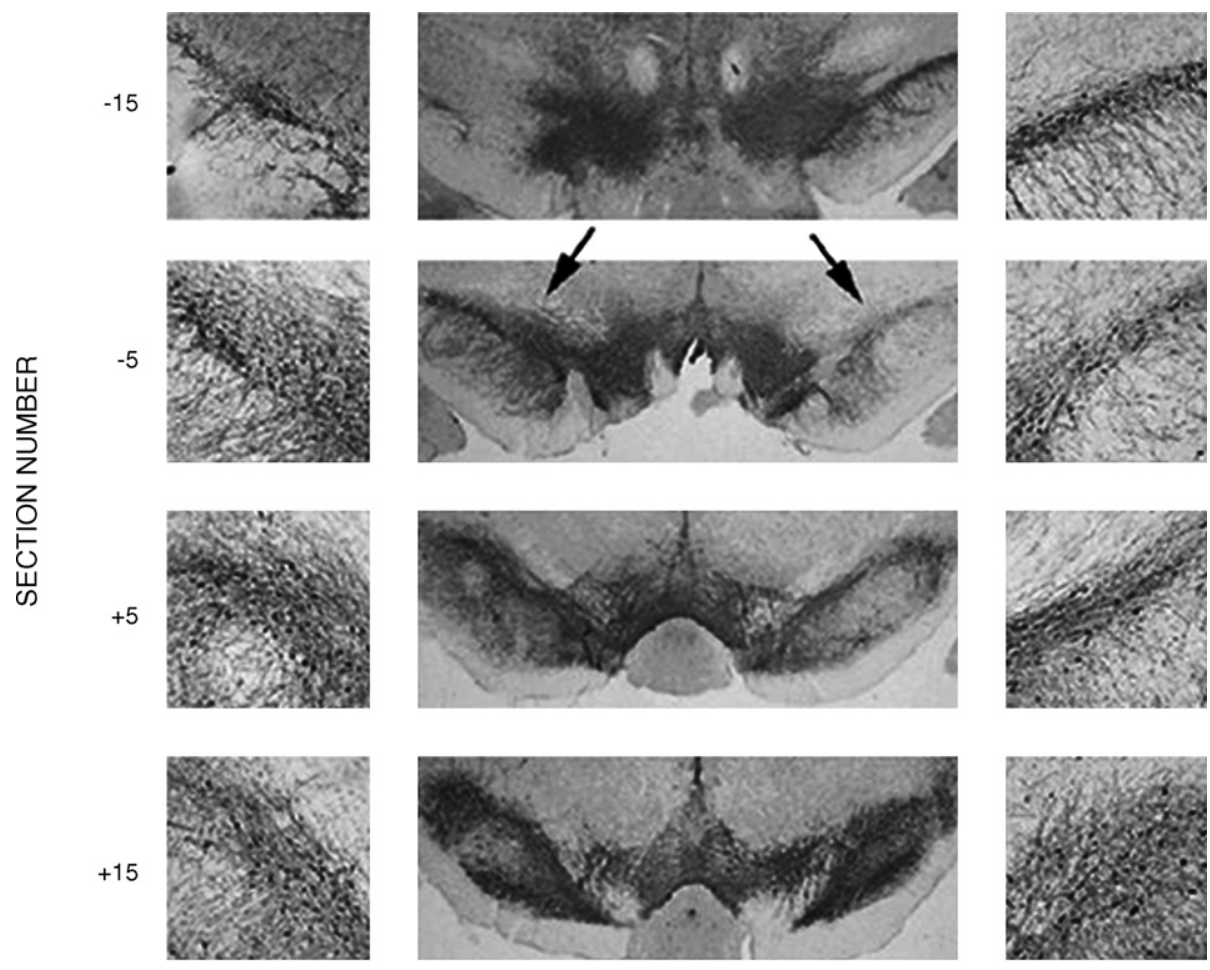

SYN

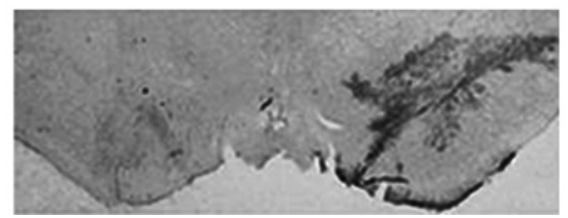

Fig. 5. Immunohistochemical analysis of the $\mathrm{SN}$ of a rat after unilateral injection with $\mathrm{LV}-\alpha-\mathrm{SYN}(\mathrm{A} 30 \mathrm{P})$. The four upper panels represent $\mathrm{TH}$ immunostainings of serial sections with an interval of $500 \mu \mathrm{m}$ around the injection site. The panels on the side represent a higher magnification of the dopaminergic neurons of the substantia nigra. The lowest panel is a section stained with an antibody against $\alpha$-synuclein demonstrating the unilateral overexpression of the protein. In Section 5 there is an almost complete loss of dopaminergic neurons (arrows). Histological analysis was performed 46 weeks after injection.

In the LV- $\alpha-\mathrm{SYN}(\mathrm{A} 30 \mathrm{P})$ injected animals, there was a consistent reduction in the number of dopamine transporters in the striatum of the injected hemisphere (Fig. 2b). From the five $L V-\alpha-S Y N(A 30 P$ )-transduced rats that were analyzed by microSPECT, two rats had received a contralateral injection with GFP-expressing LV and one rat an injection with saline to exclude injection artifacts.

Fig. 3 shows the right-to-left binding asymmetry index as a measure of relative dopamine transporter activity compared to the unaffected side for the three groups. The intersubject variability of asymmetry ratios was $13 \%$ in normal Wistar rats. LV-SYN(A30P)-transduced rats showed up to $31 \%$ reduction in dopamine transporter binding (mean $10 \pm 15 \%$, $p<0.05$ ) compared to normal rats, irrespective of contralateral control injections.

\subsection{Histological analysis}

After the microSPECT measurement, rats were sacrificed for histological analysis. In the substantia nigra, every fifth section of the brain was stained for $\mathrm{TH}$ and quantified by stereological procedures (Fig. 4). In a number of animals, loss of dopaminergic neurons was apparent in sections adjacent to the injection site, (Fig. 5). As a control, we also evaluated normal, non-injected rats $(n=4)$; in these rats no asymmetry in the number of dopaminergic neurons between the two hemispheres was observed. Correlation analysis between the dopaminergic neuron loss obtained by histological quantification and the loss of dopamine transporters $(R / L$ binding asymmetry) visualized by microSPECT (Fig. 6) showed a good correlation $\left(R^{2}=0.84, p=0.00003\right)$.

We subsequently analyzed the rats that did not display asymmetric behavior in the amphetamine-induced rotational test. There was a small but non-significant reduction $(p=0.11)$ in the ratio of dopaminergic neurons between the injected side and the contralateral side (Fig. 4). Thus, the extent of dopaminergic neuron loss was different between the $\alpha$-synuclein-transduced rats that showed asymmetric rotational behavior in the amphetamine-induced rotation test and the group that remained negative or the control group. In the 


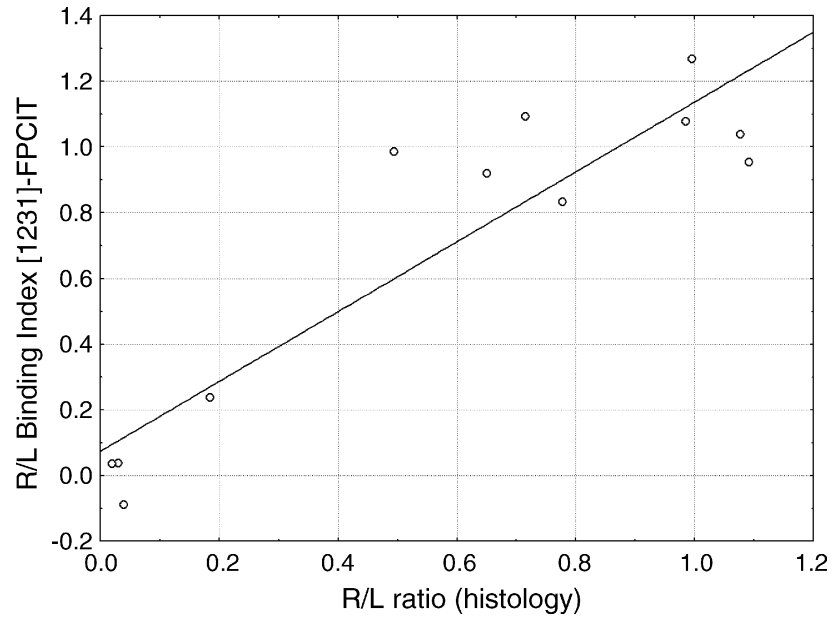

Fig. 6. Correlation between the dopaminergic cell loss visualized by histological analysis and the reduction of dopamine transporters visualized by SPECT analysis in the rats that were positive in the rotation test.

6OHDA rats we observed an average unilateral destruction of $93 \%$ of the nigral dopaminergic neurons. In the LV- $\alpha-$ SYN(A30P) injected rats, we observed a clear reduction of dopaminergic neurons in the injected hemisphere that varied between 22 and $51 \%(n=4, p<0.05)$ (Fig. 4)
Further morphological analysis revealed $\alpha$-synucleininduced pathology in the surviving dopaminergic neurons of the substantia nigra. These pathological changes were similar to what we observed after LV-mediated overexpression of $\alpha$ synuclein in the mouse brain [20], i.e. $\alpha$-synuclein-containing inclusions in the cytoplasm (Fig. 7a) and neuritic enlargements in the striatum (Fig. 7b). A fraction of these inclusions were ubiquitin-positive (Fig. 7, lower panels), also in agreement with our previous results.

\section{Discussion}

We have previously demonstrated $\alpha$-synuclein-induced pathology in the mouse brain [20]. Here we report a longterm study on the effects of LV-mediated overexpression of $\alpha$-synuclein in the $\mathrm{SN}$ of the rat. Next to examining neuropathology, the aim of this study was to validate this animal model by behavioral testing and non-invasive molecular imaging.

While this work was ongoing, other groups have reported as well on viral vector-mediated overexpression of $\alpha$ synuclein in the substantia nigra of the rat $[6,17,18]$ or the monkey [15]. Klein et al. monitored rats over a time course of 1 year after adeno-associated virus vector-mediated expres-
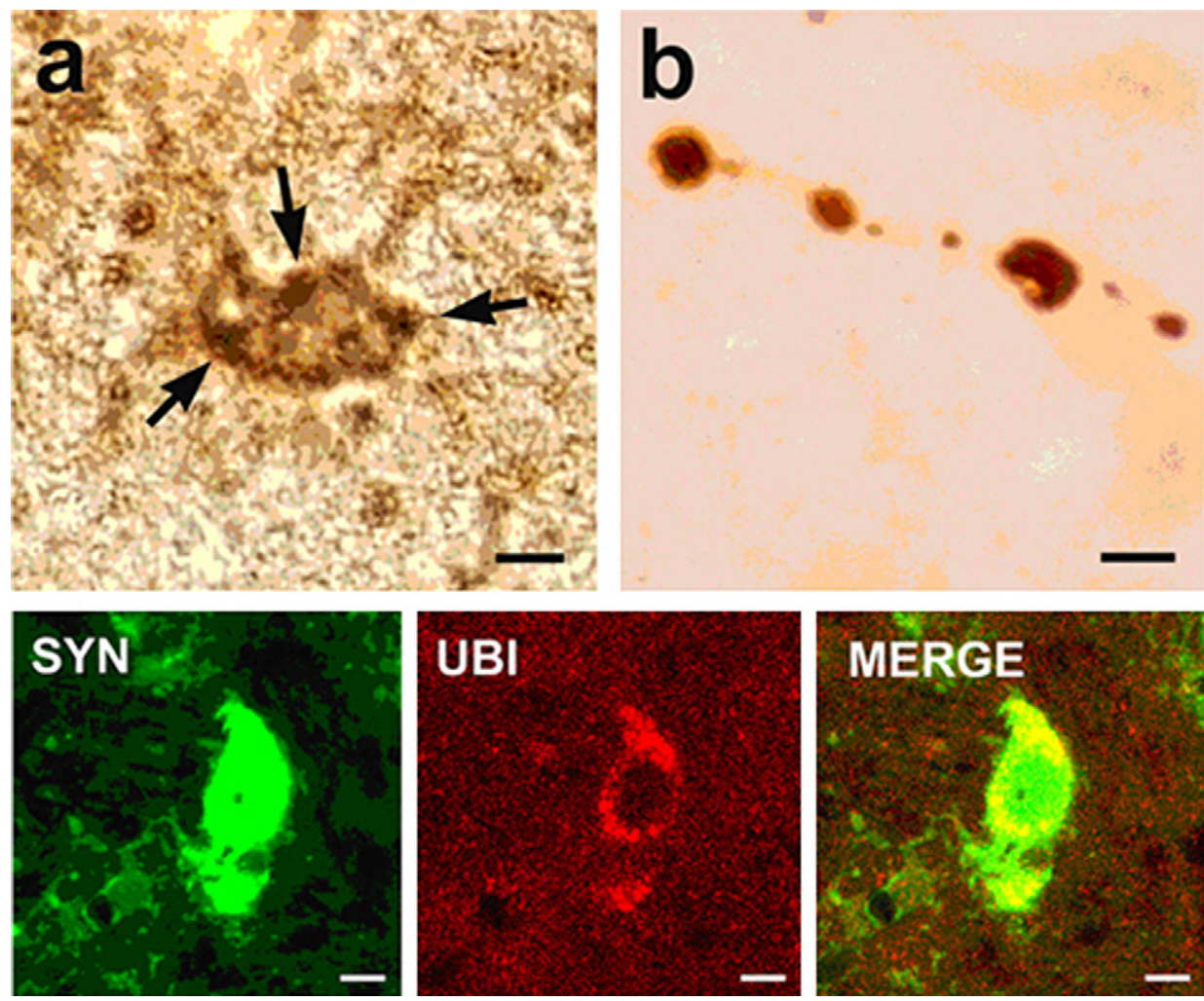

Fig. 7. $\alpha$-Synuclein-induced neuropathological changes. Immunohistochemical stainings for $\alpha$-synuclein demonstrate: (a) cytoplasmic $\alpha$-synuclein-containing inclusions in the substantia nigra and (b) $\alpha$-synuclein-containing neuritic inclusions in the striatum. The arrows indicate the presence of the cytoplasmic inclusions. The lower panels represent fluorescent double-stainings for $\alpha$-synuclein and ubiquitin analysed by confocal microscopy demonstrating the presence of ubiquitin-containing inclusions in transduced neurons. Scale bars represent $10 \mu \mathrm{m}$ in the upper panels and $5 \mu \mathrm{m}$ in the lower panels. 
sion of A30P $\alpha$-synuclein [18]. Apart from Lewy-like inclusions of $\alpha$-synuclein and dystrophic neurites, no abnormal behavior or motor dysfunction was found in either rotational or rotating rod testing despite of a 53\% loss of dopaminergic neurons. In contrast, after AAV-mediated overexpression of wild type and A53T mutant $\alpha$-synuclein in the substantia nigra of the rat, significant motor impairment was observed in a subgroup of animals in which dopaminergic neuron cell loss exceeded a critical threshold of 50-60\% [17]. The rats were monitored for up to 6 months. Interestingly, a maximal effect was observed at 8 weeks. At 8 months, there was a recovery of the total number of nigral $\mathrm{TH}+$ cells and striatal $\mathrm{TH}+$ fibers. However, striatal dopamine levels were still reduced by $50 \%$ [17]. In line with this observation, the rats showed impaired skilled paw use at 4 and 5 months after vector injection. Dopaminergic cell loss was also observed after LV-mediated overexpression of different forms of human but not rat $\alpha$-synuclein in the substantia nigra of the rat [6]. This study reported no behavioral changes.

We have examined motor function at different time points after injection of LV encoding A30P $\alpha$-synuclein in the substantia nigra of the rat. There was no aberrant behavior at any time point in the stepping test, while the number of amphetamine-induced rotations varied considerably between individual rats. Some rats displayed a relatively high number of ipsiversive rotations while other rats were not or very mildly affected. These findings are in agreement with the results of Kirik et al. [17] where about $25 \%$ of the animals were significantly impaired. Previous studies have shown that significant changes in amphetamine-induced behavior are only detected when the number of dopaminergic neurons is reduced by $50-60 \%$ [21]. In our study we have included two groups of rats that differed only in the levels of A30P $\alpha$-synuclein expression. Interestingly, in the group expressing modest levels of $\alpha$-synuclein, fewer rats were positive in the amphetamine-induced rotation test, and the impairment started at a later time point. In the high expression group, there was an apparent reversal of behavioral impairment at the latest time point (Fig. 2). We speculate that in agreement with the findings of Kirik et al. (2002), the rats with higher expression levels of $\alpha$-synuclein may already have partially recovered and the TH+ fiber network may have regenerated. Those rats that showed asymmetric rotational behavior also displayed a modest reduction in dopamine transporter binding as analyzed by microSPECT. In these rats dopaminergic cell counts in the substantia nigra revealed a cell loss between 20 and 50\%. Further immunohistochemical analysis of the rats that behaved normal in the rotation test demonstrated a correlation between motor behavior and dopaminergic cell death. The percentage of dopaminergic cell death in our rats is lower than that reported by other groups. Differences between studies may relate to the use of AAV vectors $[17,18]$ versus $\mathrm{LV}$ vectors or the use of different promoters (PGK versus CMV) [6].

Imaging of dopamine transporters by SPECT is an important tool for the study of the integrity of nigrostriatal dopamin- ergic nerve terminals in vivo in humans [8] or in animal models [28]. In Parkinson's disease there is a loss of the presynaptic dopamine transporters in the striatum as a result of the degeneration of the dopaminergic neurons of the substantia nigra. In humans, the sensitivity of this technique allows assessment of the loss of dopamine transporters in the earliest phase of PD [12,28,29] and even before the onset of motor symptoms [5]. The technique is used in individual patients to corroborate the diagnosis, to differentiate patients with presynaptic Parkinsonism from other forms of Parkinsonism (such as essential tremor and medication-induced Parkinsonism) [7], to assess progression of degeneration and to evaluate neuroprotective strategies $[25,33]$.

From our results on the intersubject variability and the extent of the demonstrated deficit in rats injected with $\alpha-S Y N ~ L V$, it can be expected that we can monitor $\alpha$ synuclein-induced neurodegeneration of the nigro-striatal system by microSPECT. Non-invasive imaging of disease progression in animal models would be very advantageous because it allows individual kinetic follow-up and circumvents interindividual variability. All the rats that were selected based on impaired amphetamine-induced behavior showed reduced dopamine transporter binding by microSPECT. Therefore, imaging of the rat brain by microSPECT is expected to be a sensitive non-invasive technique to monitor limited and longitudinal degeneration of the dopaminergic neurons in the substantia nigra. We are planning microSPECT analysis at different time points after stereotactic delivery of lentiviral vectors to monitor the kinetics of $\alpha$-synucleininduced dysfunction of the dopaminergic system in individual rats. From the methodological side, the absence of partial-volume correction for the limited resolution of the microSPECT measurement may have caused the underestimation of the true dopaminergic deficit by around $10 \%$ when compared to the histological dopaminergic neuron counting. To resolve this quantification issue, microMRI (magnetic resonance imaging) investigations can be performed and post hoc or reconstruction-based resolution recovery techniques could then be applied [2].

In conclusion, our results provide a proof-of-principle for a rat model for early PD by LV-mediated overexpression of $\alpha$-SYN in the substantia nigra. Validation of the model was performed by both behavioral testing and by non-invasive microSPECT imaging. To our knowledge our results provide the first example of non-invasive imaging of chronicprogressive neurodegeneration in a genetic rodent model of PD. We believe that this novel rat model will facilitate the development of drugs targeting $\alpha$-SYN aggregation as a novel strategy for the treatment of PD.

\section{Acknowledgements}

We are very grateful to D. Trono (University of Geneva, Switzerland) for receiving HIV vector constructs, K. Conway (Center for Neurologic Diseases, Boston) for $\alpha$-synuclein 
cDNA. We acknowledge excellent technical assistance from S. Willems, M. Michiels, L. Luyts, A. Van der Perren, P. Vermaelen and B. Vanbilloen.

VB is a postdoctoral fellow of the Flemish Fund for Scientific Research (FWO Vlaanderen). KVL is supported by a Clinical Research Mandate of the Flemish Fund for Scientific Research. This work was supported by the K.U. Leuven (IDO 01/004, IDO/02/012), the FWO Vlaanderen (G.0164.03), the Institute for the Promotion of Innovation by Science and Technology in Flanders (IWT SBO/030238) and the European Community (NEUROPARK, QLK3-CT-200202114).

Disclosure statement: the authors declare no conflicts of interest.

\section{References}

[1] Baekelandt V, Claeys A, Eggermont K, et al. Characterization of lentiviral vector-mediated gene transfer in adult mouse brain. Hum Gene Ther 2002;13(7):841-53.

[2] Baete K, Nuyts J, Van LK, et al. Evaluation of anatomy based reconstruction for partial volume correction in brain FDG-PET. Neuroimage 2004;23(1):305-17.

[3] Beal MF. Experimental models of Parkinson's disease. Nat Rev Neurosci 2001;2(5):325-34.

[4] Beque D, Nuyts J, Bormans G, Suetens P, Dupont P. Characterization of pinhole SPECT acquisition geometry. IEEE Trans Med Imaging 2003;22(5):599-612.

[5] Berendse HW, Booij J, Francot CM, et al. Subclinical dopaminergic dysfunction in asymptomatic Parkinson's disease patients' relatives with a decreased sense of smell. Ann Neurol 2001;50(1):34-41.

[6] Bianco CL, Ridet JL, Schneider BL, Deglon N, Aebischer P. \{alpha\}Synucleinopathy and selective dopaminergic neuron loss in a rat lentiviral-based model of Parkinson's disease. Proc Natl Acad Sci USA 2002;99(16):10813-8.

[7] Booij J, Speelman JD, Horstink MW, Wolters EC. The clinical benefit of imaging striatal dopamine transporters with [123I]FP-CIT SPET in differentiating patients with presynaptic Parkinsonism from those with other forms of Parkinsonism. Eur J Nucl Med 2001;28(3):266-72.

[8] Booij J, Tissingh G, Winogrodzka A, van Royen EA. Imaging of the dopaminergic neurotransmission system using single-photon emission tomography and positron emission tomography in patients with Parkinsonism. Eur J Nucl Med 1999;26(2):171-82.

[9] Conway KA, Harper JD, Lansbury PT. Accelerated in vitro fibril formation by a mutant alpha-synuclein linked to early-onset Parkinson disease. Nat Med 1998;4(11):1318-20.

[10] Feany MB, Bender WW. A Drosophila model of Parkinson's disease. Nature 2000;404(6776):394-8.

[11] Follenzi A, Ailles LE, Bakovic S, Geuna M, Naldini L. Gene transfer by lentiviral vectors is limited by nuclear translocation and rescued by HIV-1 pol sequences. Nat Genet 2000;25(2):217-22.

[12] Frost JJ. Receptor imaging by positron emission tomography and single-photon emission computed tomography. Invest Radiol 1992;27(Suppl 2):S54-8.

[13] Giasson BI, Uryu K, Trojanowski JQ, Lee VM. Mutant and wild type human alpha-synucleins assemble into elongated filaments with distinct morphologies in vitro. J Biol Chem 1999;274(12):761922.

[14] Kahle PJ, Neumann M, Ozmen L, et al. Subcellular localization of wild-type and Parkinson's disease-associated mutant alpha-synuclein in human and transgenic mouse brain. J Neurosci 2000;20(17): $6365-73$.
[15] Kirik D, Annett LE, Burger C, Muzyczka N, Mandel RJ, Bjorklund A. Nigrostriatal $\{$ alpha\}-synucleinopathy induced by viral vectormediated overexpression of human $\{$ alpha $\}$-synuclein: a new primate model of Parkinson's disease. Proc Natl Acad Sci USA 2003.

[16] Kirik D, Rosenblad C, Bjorklund A. Characterization of behavioral and neurodegenerative changes following partial lesions of the nigrostriatal dopamine system induced by intrastriatal 6-hydroxydopamine in the rat. Exp Neurol 1998;152(2):259-77.

[17] Kirik D, Rosenblad C, Burger C, et al. Parkinson-like neurodegeneration induced by targeted overexpression of alpha-synuclein in the nigrostriatal system. J Neurosci 2002;22(7):2780-91.

[18] Klein RL, King MA, Hamby ME, Meyer EM. Dopaminergic cell loss induced by human $\mathrm{A} 30 \mathrm{P}$ alpha-synuclein gene transfer to the rat substantia nigra. Hum Gene Ther 2002;13(5):605-12.

[19] Kruger R, Kuhn W, Muller T, et al. Ala30Pro mutation in the gene encoding alpha-synuclein in Parkinson's disease. Nat Genet 1998;18(2):106-8.

[20] Lauwers E, Debyser Z, van Dorpe J, De Strooper B, Nuttin B, Baekelandt $V$. Neuropathology and neurodegeneration in rodent brain induced by lentiviral vector-mediated overexpression of alpha-synuclein. Brain Pathol 2003;13(3):364-72.

[21] Lee CS, Sauer H, Bjorklund A. Dopaminergic neuronal degeneration and motor impairments following axon terminal lesion by instrastriatal 6-hydroxydopamine in the rat. Neuroscience 1996;72(3):641-53.

[22] Masliah E, Rockenstein E, Veinbergs I, et al. Dopaminergic loss and inclusion body formation in alpha-synuclein mice: implications for neurodegenerative disorders. Science 2000;287(5456):1265-9.

[23] Naldini L, Blömer U, Gallay P, et al. In vivo gene delivery and stable transduction of nondividing cells by a lentiviral vector. Science 1996;272(5259):263-7.

[24] Perese DA, Ulman J, Viola J, Ewing SE, Bankiewicz KS. A 6hydroxydopamine-induced selective Parkinsonian rat model. Brain Res 1989;494(2):285-93.

[25] Pirker W, Djamshidian S, Asenbaum S, et al. Progression of dopaminergic degeneration in Parkinson's disease and atypical Parkinsonism: a longitudinal beta-CIT SPECT study. Movement Disord 2002;17(1):45-53.

[26] Polymeropoulos MH, Lavedan C, Leroy E, et al. Mutation in the alphasynuclein gene identified in families with Parkinson's disease. Science 1997;276(5321):2045-7.

[27] Richfield EK, Thiruchelvam MJ, Cory-Slechta DA, et al. Behavioral and neurochemical effects of wild-type and mutated human alphasynuclein in transgenic mice. Exp Neurol 2002;175(1):35-48.

[28] Scherfler C, Donnemiller E, Schocke M, et al. Evaluation of striatal dopamine transporter function in rats by in vivo beta-[123I]CIT pinhole SPECT. Neuroimage 2002;17(1):128-41.

[29] Seibyl JP, Marek KL, Quinlan D, et al. Decreased single-photon emission computed tomographic [123I]beta-CIT striatal uptake correlates with symptom severity in Parkinson's disease. Ann Neurol 1995;38(4):589-98.

[30] Spillantini MG, Schmidt ML, Lee VM, Trojanowski JQ, Jakes R, Goedert M. Alpha-synuclein in Lewy bodies. Nature 1997;388(6645):839-40.

[31] Ungerstedt U. Striatal dopamine release after amphetamine or nerve degeneration revealed by rotational behaviour. Acta Physiol Scand Suppl 1971;367:49-68.

[32] van Der Putten H, Wiederhold KH, Probst A, et al. Neuropathology in mice expressing human alpha-synuclein. J Neurosci 2000;20(16):6021-9.

[33] Winogrodzka A, Bergmans P, Booij J, van Royen EA, Janssen AG, Wolters EC. [123I]FP-CIT SPECT is a useful method to monitor the rate of dopaminergic degeneration in early-stage Parkinson's disease. J Neural Transm 2001;108(8-9):1011-9.

[34] Zarranz JJ, Alegre J, Gomez-Esteban JC, et al. The new mutation, E46K, of alpha-synuclein causes Parkinson and Lewy body dementia. Ann Neurol 2004;55(2):164-73. 
[35] Zennou V, Petit C, Guetard D, Nerhbass U, Montagnier L, Charneau P. HIV-1 genome nuclear import is mediated by a central DNA flap. Cell 2000;101(2):173-85.

[36] Zufferey R, Donello JE, Trono D, Hope TJ. Woodchuck hepatitis virus posttranscriptional regulatory element enhances expression of transgenes delivered by retroviral vectors. J Virol 1999;73(4): 2886-92.

[37] Zufferey R, Dull T, Mandel RJ, et al. Self-inactivating lentivirus vector for safe and efficient in vivo gene delivery. J Virol 1998;72(12):9873-80. 